\title{
SUITOR: selecting the number of mutational signatures through cross-validation
}

Donghyuk Lee

National Cancer Institute

Difei Wang

National Cancer Institute

Xiaohong Yang

National Cancer Institute, National Institutes of Health

Jianxin Shi

National Cancer Institute

Maria Teresa Landi

National Cancer Institute https://orcid.org/0000-0003-4507-329X

Bin Zhu ( $\nabla$ bin.zhu@nih.gov )

National Cancer Institute https://orcid.org/0000-0003-0172-5516

\section{Article}

Keywords: SUITOR, cancer, genome sequenced tumors, mutational signatures

Posted Date: September 8th, 2020

DOI: https://doi.org/10.21203/rs.3.rs-67930/v1

License: (c) (i) This work is licensed under a Creative Commons Attribution 4.0 International License. Read Full License

Version of Record: A version of this preprint was published at PLOS Computational Biology on April 4th, 2022. See the published version at https://doi.org/10.1371/journal.pcbi.1009309. 


\title{
SUITOR: selecting the number of mutational signatures through cross-validation
}

Donghyuk Lee ${ }^{1}$, Difei Wang ${ }^{1}$, Xiaohong R. Yang ${ }^{1}$, Jianxin Shi ${ }^{1}$, Maria Teresa Landi $^{1}$, Bin Zhu ${ }^{1, \#}$ ${ }^{1}$ Division of Cancer Epidemiology and Genetics, National Cancer Institute, National Institutes of Health, Bethesda, MD, USA

\# To whom correspondence should be addressed to: bin.zhu@nih.gov

\begin{abstract}
For de novo mutational signature analysis, the critical first step is to decide how many signatures should be expected in a cancer genomics study. An incorrect number could mislead downstream analyses. Here we present SUITOR (selecting the number of mutational signatures through cross-validation), an unsupervised cross-validation method that requires little assumptions and no numerical approximations to select the optimal number of signatures without overfitting the data. In vitro studies and in silico simulations demonstrated that SUITOR can correctly identify signatures, some of which were missed by other widely used methods. Applied to 1,536 whole-genome sequenced tumors across eight cancer types, SUITOR selected signatures with the smallest prediction errors and almost all signatures of breast cancer selected by SUITOR were validated in an independent study. SUITOR is a powerful tool to select the optimal number of mutational signatures, facilitating downstream analyses with etiological or therapeutic importance.
\end{abstract}




\section{Background}

Mutational signatures are patterns of somatic mutations imprinted on the cancer genome by operative mutational processes. At least 49 single base substitution mutational signatures have been identified across cancer types ${ }^{1}$, with some associated with exogenous mutagenic exposures $^{2-4}$ and endogenous mutational processes ${ }^{5-8}$. Moreover, mutational signatures have been proposed to identify cancer predisposition genes (e.g., NTHL1 in breast cancer ${ }^{9}$ ), and to stratify cancer patients ${ }^{10-13}$ for precision treatment. In these studies, deciding the expected number of signatures is the pivotal first step, which determines the downstream steps of extracting signature profiles, estimating signature contributions and stratifying tumors based on signatures for treatment.

Despite the many algorithms proposed to extract signature profiles ${ }^{14-18}$ and estimate signature contributions ${ }^{19,20}$, relatively little emphasis has been placed on selecting the correct number of de novo mutational signatures in cancer genomics studies ${ }^{21,22}$. SomaticSignatures ${ }^{14}$ chooses the number of signatures based on the residual sum of squares and the explained variance without a clearly defined selection criterion. SigProfiler ${ }^{15}$ considers the mean reconstruction error and the stability of signature extraction; however, it is unclear how these features could be combined to jointly predict the number of signatures. $\mathrm{EMu}^{16}$ and signe ${ }^{17}$ adopt a Bayesian information criterion $(\mathrm{BIC})^{23}$. Although $\mathrm{BIC}$ is a popular model selection criterion for supervised learning (e.g., regression and classification) where the number of parameters is fixed, it may not be applicable to unsupervised learning, including mutational signature analysis, where the number of parameters increases with the sample size (other limitations of BIC elaborated in 
Supplementary Note 1). SignatureAnalyzer ${ }^{1}$ uses an automatic relevance determination (ARD) prior $^{24}$ which imposes sparsity on mutation profiles and contributions. The number of signatures chosen by signatureAnalyzer is sensitive to the pre-specified hyperparameters of the ARD prior and the tolerance level.

To overcome the limitations of previous methods, we propose selecting the number of signatures through cross-validation. Unlike the BIC or the ARD prior, cross-validation requires little assumptions and no numerical approximations ${ }^{25}$. Additionally, it is intuitive to appreciate the benefits of a cross-validation approach: if the full dataset is split into a training set and a validation set, the "correct" set of signatures discovered in the training set should predict the validation data well.

Despite being conceptually appealing, the standard cross-validation approach does not work for unsupervised mutational signature analysis. In the standard cross-validation scheme, it is feasible to remove a subset of subjects all together as a validation set because parameters (e.g., regression coefficients) are identical across subjects. However, mutation contributions as parameters are tumor-specific in mutational signature analysis. If mutation counts of a subset of tumors were removed all together as a validation set, these counts would be used twice (for estimating tumor-specific mutation contributions and calculating the prediction error), which would underestimate the prediction error. Instead, cross-validation for mutational signature analysis requires retaining all tumors in the training set but removing some mutation counts 
from each tumor as a validation set. Consequently, missing data emerge in the training set, which would cause current methods for mutational signature analysis to fail.

Here we present SUITOR (selecting the number of mutational signatures through crossvalidation), an unsupervised cross-validation method that selects the optimal number of signatures to attain the minimal prediction error in the validation set. SUITOR extends the probabilistic model to allow missing data in the training set, which makes cross-validation

feasible. Moreover, we propose an expectation/conditional maximization (ECM) algorithm ${ }^{26}$ to extract signature profiles, estimate mutation contributions and impute the missing data simultaneously. We demonstrated SUITOR's superior performance using in vitro experimental data, in silico simulations, in vivo applications to 1,536 tumors across eight cancer types, and validation of signatures of breast cancer in additional 440 breast tumors.

\section{Results}

\section{Overview of SUITOR}

SUITOR is built upon a probabilistic mode ${ }^{27,28}$, for which the maximum likelihood estimation (MLE) is equivalent to the solution of non-negative matrix factorization (NMF), the most popular method for mutational signature analysis (details in Methods). The steps of SUTOR are outlined as follows (Fig.1 with details in Methods): we first separate the mutation catalog matrix into the training and validation sets. The training set contains missing data held out as validation data. Next, following an initial step, the ECM algorithm iteratively imputes the missing data in the E-step (expectation step) and estimates the signature contributions (of the 
W matrix) and profiles (of the $\mathrm{H}$ matrix) in the CM-steps (conditional maximization steps) till coverage. Finally, the missing data are imputed and compared to the validation data to calculate the prediction error in the validation set. These steps are conducted for multiple candidate numbers of signatures and the one with the minimal prediction error will be chosen as the optimal number of signatures.

Notably, it's critical to evaluate the prediction error in the validation set instead of the training set. The prediction error in the training set will keep reducing with more signatures, while the prediction error in the validation set will decrease first and then inflate, as illustrated by prediction error curves in Fig. 1, due to the well-known bias-variance tradeoff for model complexity ${ }^{29}$ measured by the number of signatures. Hence, the number of signatures selected by SUITOR with the minimal prediction error in the validation set corresponds to the optimal bias-variance tradeoff.

\section{Evaluation of SUITOR in two in vitro studies}

We assessed the performance of SUITOR in two experimental studies ${ }^{2,5}$, for which the true number and profile of signatures were generated experimentally and validated in vitro (see details in Methods). The first study created endogenous mutational signatures through CRISPRCas9-mediated knockouts of DNA repair genes in an isogenic human cell line ${ }^{5}$. The second study generated exogenous mutational signatures in human-induced pluripotent stem cell (iPSC) lines exposed to environmental mutagens ${ }^{2}$. For both studies, we evaluated whether SUITOR could correctly select the number of signatures and recover the profiles of single base substitution 
(SBS) signatures. We then compared SUITOR's performance with sigProfiler, signatureAnalyzer and signeR.

For the MSH6 knockout study, SUITOR correctly selected the background and MSH6 knockoutinduced signatures (Fig. 2a) and recovered the corresponding signature profiles (Fig. 2b; Supplementary Table 1). SigProfiler, signatureAnalyzer and signeR correctly identified these two signatures as well (Supplementary Fig. 1; Supplementary Table 1). Next, we extracted signatures of knock-out studies of six DNA repair genes (CHK2, NEIL1, NUDT1, POLB, POLE and POLM), which did not induce experimentally detectable signatures ${ }^{5}$. SUITOR and the other three methods correctly identified one background signature only with no additional signatures (Supplementary Fig. 2). We conclude that in this in vitro study with at most two signatures, all four methods perform equally well. This is not the case when the number of signatures increases as shown below.

We tested four methods for retrieving nine mutagen-induced signatures in iPSC lines. SUITOR correctly discovered one background and eight mutagen-induced signatures (Fig. 2c) with profiles closely resembling the true in vitro signatures (cosine similarity range: 0.83-0.99; Supplementary Table 2). In contrast, sigProfiler, signatureAnalyzer and signeR missed few signatures (Supplementary Fig. 3; Supplementary Table 2), which would impair the stratification of tumor subclones based on signature contributions. Indeed, sigProfiler and signeR merged subclones exposed to 1,8-Dinitropyrene (1,8-DNP) with subclones exposed to 2-amino-1methyl-6-phenylimidazo[4,5-b]pyridine (PhIP); while signatureAnalyzer grouped two subclones 
exposed to PhIP with controls which were not exposed to mutagens (Fig. 2d). In contrast, SUITOR clearly separated subclones into distinct clusters, each corresponding to a unique mutagen exposure. Note that such separated cluster pattern is unlikely driven by sequencing artifacts, because a) the DNA samples derived from the subclones with different mutagen exposures were pooled and sequenced together and b) all identified signatures were matched to either known background or mutagen-induced signatures with no unrecognized signatures likely due to sequencing artifacts.

\section{In silico simulation studies}

We evaluated SUITOR and three other methods through additional in silico simulations. We first considered a setting of one true signature, for which any additional signatures found would be false positives (details in Methods). SUITOR, sigProfiler and signeR correctly identified the single true signature (Supplementary Fig. 4) in 20 of 20 replicates; signatureAnalyzer found a false positive signature (Fig. 3a) in 17 of 20 replicates. Next, we examined a setting of nine true signatures with varying signature contributions (details in Methods; Supplementary Fig. 5; Supplementary Table 3). All methods correctly identified six common signatures (SBS1, 2, 3, 5, $13,18)$ in 20 of 20 replicates; SUITOR and signatureAnalyzer detected two rare signatures $(\mathrm{SBS} 8,41)$ in more replicates than the other two methods (Fig. 3b). None of the methods identified the extremely rare and flat signature SBS40 (present in one tumor only). Notably, all methods did not detect other signatures besides nine true signatures. Together, the simulation studies suggest that SUITOR is able to find both common and rare signatures while well controlling the rate of false positives. 


\section{PCAWG mutational signatures of eight cancer types}

We tested the four methods in whole-genome sequencing (WGS) data of 1,536 tumors across eight cancer types (each with at least 100 tumors) from the Pan-Cancer Analysis of Whole Genomes (PCAWG) study ${ }^{1}$ (details in Methods).

First, we extracted de novo mutation signatures for one cancer type at a time. Unlike in vitro or in silico studies, the true signatures were unknown here. Nevertheless, we could evaluate if the signatures detected in part of the dataset predict mutation counts in the remaining part well. Specially, the mutation catalog matrix will be separated into training, validation and testing sets, the last of which is used to evaluate performance of the selected number of signatures (details in Methods). Among four methods, SUITOR clearly attained the smallest prediction errors across all cancer types (Fig. 4a). Moreover, most signatures found by SUITOR were highly similar to the COSMIC signatures (with cosine similarity $>0.8$, Fig. $4 \mathrm{~b}$ ) and frequently detected by other methods (Fig. 4c). In contrast, signatureAnalyzer identified more de novo signatures, some of which were not matched to any COSMIC signatures (Fig. 4b).

Next, we extracted de novo signatures combining WGS data of 1,539 tumors. SUITOR found 16 signatures, all of which could be matched to the COSMIC signatures (Fig. 4b). These signatures had the smallest prediction error, compared to signatures detected by the other methods (Fig. 4a). As expected, the signatures commonly found in multiple cancer types (e.g., SBS1, SBS2 and SBS13) could be identified when combining all cancer types together, while signatures specific 
to a single cancer type (e.g., SBS24 specific to liver cancer) were absent in the combined signature analysis (Fig. 4c). Notably, five clusters emerged when we clustered 1,539 tumors using signature contributions estimated by SUITOR (Fig. 5a). Liver tumors and kidney tumors formed two distinct clusters, possibly due to the signatures specific to these two cancer types respectively; the remaining clusters included: i) almost all ovarian tumors and a subset of breast, pancreatic and prostate tumors; ii) most of lymphomas and few pancreatic tumors; iii) all remaining tumors. SigAnalyzer and signeR showed similar five clusters, but less so sigProfiler (Fig. 5b-d). Such distinctive patterns confirm that signatures could possibly indicate etiological differences and help identify the tissue of origin in tumors of unknown primary ${ }^{30}$.

\section{External validation of breast cancer mutational signatures}

We validated PCAWG breast cancer signatures (based on 194 breast tumors) using an independent WGS set of 440 breast tumors of the Sanger breast cancer (BRCA) study from the same race population ${ }^{31}$. SUITOR (Fig. 6a and Supplementary Table 4) and signeR (Supplemental Table 5) identified nine breast cancer signatures in PCAWG and validated eight in the Sanger BRCA study (with cosine similarity $>0.8$ ); sigProfiler identified eight signatures and confirmed seven (Supplementary Table 6); signatureAnalyzer identified twelve and validated eight (Supplementary Table 7). Overall six signatures (SBS1,2,8,13,17b and 18) were identified in both studies by all methods, while the flat featureless signature SBS5 could not be validated by any method. 
Besides validating eight PCAWG breast cancer signatures, SUITOR found four additional signatures in the Sanger BRCA study (Fig. 6a and Supplementary Table 8). Two of them were highly similar to COSMIC signatures SBS26 and SBS30 (cosine similarity $>0.93$ ) and were identified by the other three methods as well; the other two (similar to SBS3 and SBS6) were also detected by signatureAnalyzer and/or signeR. Similarly, three other methods found few more signatures as well (Supplementary Tables 9-11). These findings suggest that there likely exist additional signatures in the Sanger BRCA study that are missed in the PCAWG breast cancer study because of either the larger sample size or specific operative mutational processes (e.g., BRCA1 mutation carriers with signature SBS3) in Sanger BRCA study.

Finally, as an example of clinical utility of the signatures estimated by SUITOR, we stratified the 440 breast tumors of the Sanger BRCA study using the signature contributions. Four signature clusters were found; two dominant clusters (G3 and G4) included overall 430 tumors (Fig. 6b). Compared to the G4 subgroup, the G3 subgroup showed significantly higher contributions of the nine signatures (Supplementary Fig. 6; Supplementary Table 12) and significantly lower contributions of the de novo signature A (similar to COSMIC signature SBS1 associated with aging). We found a number of clinical factors associated with the subgroups G3 and G4, including age at diagnosis and tumor grade (Supplementary Table 13). The singular most important associated factor was the molecular subtype: triple negative breast cancers were significantly enriched in the subgroup G3 (Fig. 6c; odds ratio $=25.1$, P-value $<2.2 \times 10^{-16}$, twosided Fisher's exact test). 


\section{Discussion}

It's crucial to select the correct number of de novo mutational signatures for a cancer genomics study. Here we present SUITOR that selects the number of signatures through cross-validation to minimize the prediction error in the validation set. We have shown how SUITOR outperforms common existing methods most of the time. In vitro studies show that SUITOR is capable of retrieving the correct number and profiles of both endogenous and exogenous signatures, allowing the correct stratification of tumor subclones exposed to distinct mutagens. In silico simulation studies show that SUITOR can detect common signatures in all replicates and rare signatures (as low as $1 \%$ ) in the majority of replicates. Applications to in vivo eight PCAWG cancer types show that SUITOR discovers signatures which achieve the lowest prediction errors in the testing sets. Most of these signatures are confirmed by other methods and matched to the COSMIC signatures. All except one signature found in PCAWG BRCA study were validated in the independent Sanger BRCA study. The contributions of signatures selected by SUITOR in the Sanger BRCA study are dominated by two clusters, driven by the molecular subtypes.

In addition to achieving the minimal prediction error, SUITOR enjoys other statistical and computational advantages. First, the cross-validation scheme of SUITOR guarantees that the missing data pattern does not depend on remaining or missing mutation counts in the training set. Hence, the missing data mechanism is missing completely at random (MCAR), which ensures that the estimated signature profiles and contributions would not be biased due to the missing data ${ }^{32}$. Second, the proposed ECM algorithm for SUITOR shares the convergence 
property of EM, which guarantees the increase of the likelihood function (equivalently, the decrease of generalized KL divergence) over iterations until the ECM algorithm convergences ${ }^{26}$.

Besides selecting the number of signatures of single base substitution, SUITOR could be used to select the number of signatures of other genomic alterations in tumors, including double base substitutions, small insertion and deletions (indels), and structure variations. In this paper, we used 10-fold cross validation, which was recommended as a good compromise of the biasvariance trade-off regarding the choice of $\mathrm{k}$ in $\mathrm{k}$-fold cross validation ${ }^{33,34}$. In addition, we have tried 20 -fold cross validation (i.e., $5 \%$ of data as validation data) for PCAWG studies, which lead to the same number of signatures (results were not shown).

In summary, SUITOR has shown to perform better than other methods in revealing mutational signatures, the "footprints" engraved in the cancer genomes by operative mutational processes with potentially important etiological or therapeutic implications.

\section{Methods}

\section{Non-negative matrix factorization of mutation catalog matrix}

For single base substitution (SBS), a mutation catalog matrix $\mathbf{V}$ of size $\mathrm{N} \times 96$ contains mutation counts for N cancer genomes and 96 SBS catalogs. Each SBS catalog refers to a mutated pyrimidine $(\mathrm{C}$ or $\mathrm{T})$ in the center and two unmutated adjacent nucleotides (flanking 5' and 3' bases) with total $4 \times 6 \times 4=96$ catalogs. For example, a genomic sequence $\mathrm{A} \underline{\mathrm{CG}}$ in the normal 
tissue is mutated to $\mathrm{AGG}$ in the tumor tissue. This SBS belongs to the $\mathrm{A}[\mathrm{C}>\mathrm{G}] \mathrm{G}$ mutation catalog.

Non-negative matrix factorization (NMF) is the most popular method to extract mutational signatures from the mutation catalog matrix ${ }^{15}$. Given the number of signatures $r$ to be extracted, NMF factorizes the mutation catalog matrix into two non-negative matrices: signature contribution matrix $\mathbf{W}$ of size $\mathrm{N} \times r$ and signature profile matrix $\mathbf{H}$ of size $r \times 96$ such that $\mathbf{V} \approx$ WH. Each row of $\mathbf{W}$ contains attributions of $r$ signatures, reflecting how intense $r$ mutational signatures are in a tumor; each row of $\mathbf{H}$ forms a signature profile with the elements summed to 1, showing how 96 mutation catalogs comprise a signature profile.

To estimate $\mathbf{W}$ and $\mathbf{H}$, it is common to minimize the generalized Kullback-Leibler (KL) divergence

$$
D_{K L}(\mathbf{V} \mid \mathbf{W H})=\sum_{p=1}^{96} \sum_{n=1}^{N}\left\{v_{n p} \log \left(v_{n p} / \sum_{j=1}^{r} w_{n j} h_{j p}\right)+\sum_{j=1}^{r} w_{n j} h_{j p}-v_{n p}\right\}
$$

subject to $w_{n j} \geq 0$ and $h_{j p} \geq 0$ with $1 \leq j \leq \mathrm{r}, 1 \leq n \leq \mathrm{N}$ and $1 \leq p \leq 96$. Lowercase letters, $v_{n p}, w_{n j}$ and $h_{j p}$, denote elements of the corresponding matrices, $\mathbf{V}, \mathbf{W}$ and $\mathbf{H}$, respectively. NMF can also be solved with other objective functions such as Frobenius norm or more general $\beta$-divergence, depending on the applications ${ }^{24}$.

\section{Equivalence between NMF and the solution of a probabilistic NMF model}

Notably, minimization of the generalized KL divergence is equivalent to maximize a likelihood function of a probabilistic NMF model ${ }^{28,35}$. Indeed, for a Poisson NMF model, $v_{n p}$ is assumed to be independently distributed, following a Poisson distribution with mean $\sum_{j=1}^{r} w_{n j} h_{j p}$, for the 
$n$th tumor and $p$ th mutation catalog. The log-likelihood of Poisson NMF model can be written as $\log \{\operatorname{Pr}(\mathbf{V} \mid \mathbf{W H})\}=-D_{K L}(\mathbf{V} \mid \mathbf{W H})+C$ with a constant $C$. It is clear that minimizing generalized KL divergence $D_{K L}(\mathbf{V} \mid \mathbf{W H})$ is equivalent to maximizing the $\log$-likelihood $\log \{\operatorname{Pr}(\mathbf{V} \mid \mathbf{W H})\}$. In addition, it could been shown that the multiplicative update algorithm ${ }^{36}$, which is commonly used to minimize the generalized KL divergence, is equivalent to an expectation/conditional maximization (ECM) algorithm ${ }^{26}$ for the Poisson NMF model (Supplementary Note 2). These two equivalences are used to develop SUITOR.

\section{Unsupervised cross-validation for mutational signature analysis}

SUITOR aims to select the optimal number of signatures which minimizes the prediction error in the validation set through cross-validation. Here, we describe the steps to create the validation set and challenges raised by.

For a $K$-fold cross-validation, we divide the catalog matrix $\mathbf{V}$ into $\mathrm{K}$ parts where the Poisson NMF model is fitted on $K-1$ parts as the training set, and the fitted model is validated on the remaining one part as the validation set. The cross-validation is carried out $\mathrm{K}$ times with each part served as a validation set once, using the balanced separation ${ }^{37,38}$ detailed as follows. In the $k^{t h}$ fold $(1 \leq k \leq K)$ of a balanced separation, a set of mutation counts $\left\{v_{n p} \mid p=(n \bmod 10)+\right.$ $(k-1)+a K, a=1,2, \ldots$.$\} are held out for the n^{\text {th }}$ tumor as validation data, where $a$ is restricted such that $1 \leq p \leq 96$. For example, $(1,11, \cdots, 91)^{s t}$ mutation catalogs of the first tumor are held out in the $1^{\text {st }}$ fold, $(2,12, \cdots, 92)^{\text {nd }}$ mutation catalogs in the $2^{\text {nd }}$ fold and so on. Note that the balanced separation keeps equal number of retained mutation catalogs for each tumor in the training set, which is computationally more stable than randomly splitting $\mathbf{V}$ into the 
training and validation sets. The latter may randomly remove a large number of mutation catalogs for a tumor.

As validation data are hold out, missing data emerge in the training set, because of which existing methods of NMF would fail. To address this challenge, we extend the Poisson NMF model and propose an expectation/conditional maximization (ECM) algorithm to incorporate missing data.

\section{Expectation/conditional maximization (ECM) algorithm of SUITOR}

Let $\mathcal{S}$ be the set of indices of mutation catalog matrix $\mathbf{V}$ such that $\mathcal{S}=\{(n, p) \mid 1 \leq n \leq$ $\mathrm{N}$ and $1 \leq p \leq 96\}$. For a $K$-fold cross-validation, $\mathcal{S}$ would be divided into $K$ disjoint sets $\mathcal{S}_{1}, \cdots, \mathcal{S}_{K}$. For the $k^{\text {th }}$ fold, $\mathbf{V}_{k}^{L}=\left\{v_{n p} \mid(n, p) \in \mathcal{S}_{k}\right\}$ denotes the validation set and $\mathbf{V}_{k}^{T}=$ $\left\{v_{n p} \mid(n, p) \in \mathcal{S} \backslash \mathcal{S}_{k}\right\}$ the training set, where $\mathcal{S} \backslash \mathcal{S}_{k}$ represents the indices of $\mathbf{V}$ excluding ones in $\mathcal{S}_{k}$. Mutation counts in $\mathcal{S}_{k}$ will be removed from $\mathbf{V}$ and denote as missing data $\mathbf{M}_{k}=$ $\left\{m_{n p} \mid(n, p) \in \mathcal{S}_{k}\right\}$. The $v_{n p}$ in $\mathbf{V}_{k}^{T}$ and $m_{n p}$ in $\mathbf{M}_{k}$ are assumed to be independently distributed as a Poisson distribution with the mean $\sum_{j=1}^{r} w_{n j} h_{j p}$. By the scheme of balanced separation, $\mathbf{V}_{k}^{L}$ is missing completely at random (MCAR), since $\mathbf{V}_{k}^{L}$ is removed from $\mathbf{V}$, independent of values of $\mathbf{V}_{k}^{T}$ and $\mathbf{V}_{k}^{L}$. MCAR enables us to propose an ECM algorithm to incorporate missing data and obtain unbiased estimates of $\mathbf{W}$ and $\mathbf{H}\left(\mathrm{ref}^{32}\right)$.

Next, we outline the ECM algorithm in the following iterative steps (details in Supplementary Note 3).

1. Initial step: choose initial values of $\mathbf{M}_{k}$ and set initial parameters $\mathbf{W}^{\mathbf{0}}$ and $\mathbf{H}^{\mathbf{0}}$. 
2. E-step: given the observed data $\mathbf{V}_{k}^{T}$ and the parameters $\mathbf{W}^{t}$ and $\mathbf{H}^{t}$ of the previous step $t$, the ECM algorithm calculates the conditional expectation of complete likelihood

$$
\left.Q\left(\mathbf{W}, \mathbf{H} \mid \mathbf{W}^{t}, \mathbf{H}^{t}\right)=E\left[\log \left\{\operatorname{Pr}\left(\mathbf{V}_{k}^{T}, \mathbf{M}_{k} \mid \mathbf{W} \mathbf{H}\right)\right\} \mid \mathbf{W}^{t}, \mathbf{H}^{t}\right\}\right]=-D_{K L}\left(\mathbf{V}^{*} \mid \mathbf{W H}\right)+C^{*},
$$

where $C^{*}$ is a constant independent of $\mathbf{W}$ and $\mathbf{H}, v_{n p}^{*}$ the elements of $\mathbf{V}^{*}$ as $v_{n p}^{*}=v_{n p}$ for $(n, p) \in \mathcal{S} \backslash \mathcal{S}_{k}$ and $v_{n p}^{*}=E\left[m_{n p}\right]=\sum_{j=1}^{r} w_{n j}^{t} h_{j p}^{t}$ for $(n, p) \in \mathcal{S}_{k}$.

3. CM1-step: update parameters $\mathbf{W}^{t+1}$ by maximizing $Q\left(\mathbf{W}, \mathbf{H}^{t} \mid \mathbf{W}^{t}, \mathbf{H}^{t}\right)$ with respect to $\mathbf{W}$. 4. CM2-step: update parameters $\mathbf{H}^{t+\mathbf{1}}$ by maximizing $Q\left(\mathbf{W}^{t+1}, \mathbf{H} \mid \mathbf{W}^{t}, \mathbf{H}^{t}\right)$ with respect to H.

5. Iterate steps 2 to 4 until convergence.

In the initial step, we use the median of mutation counts in $\mathbf{V}_{k}^{T}$ per each mutation catalog as initial values for $\mathbf{M}_{k}$; other more complicated methods of specifying initial values, such as nearest neighbors, lead to similar results (results not shown). We are aware that the ECM algorithm possibly converges to a local saddle point. To overcome it, we try 300 random initial values $\mathbf{W}^{\mathbf{0}}$ and $\mathbf{H}^{\mathbf{0}}$, which leads to 300 pairs of $\widehat{\boldsymbol{W}}_{i}$ and $\widehat{\boldsymbol{H}}_{i}$, the estimates of $\mathbf{W}$ and $\mathbf{H}$ for the $i$ th initial value, $i=1,2, \ldots, 300$. The final reported $\widehat{\boldsymbol{W}}$ and $\widehat{\boldsymbol{H}}$ are the $\widehat{\boldsymbol{W}}_{i}$ and $\widehat{\boldsymbol{H}}_{i}$ which maximize the $\log \left\{\operatorname{Pr}\left(\mathbf{V}_{k}^{T} \mid \widehat{\boldsymbol{W}}_{l}, \widehat{\boldsymbol{H}}_{l}\right)\right\}$ among all $\widehat{\boldsymbol{W}}_{i}^{\prime} \boldsymbol{s}$ and $\widehat{\boldsymbol{H}}_{i}{ }^{\prime} s$.

\section{Selecting number of signatures by SUITOR}


For a given number of signatures $r$, we first evaluate the prediction error, i.e., the disparity between the observed validation data $\mathbf{V}_{k}^{L}$ and the predicted ones $\widehat{\boldsymbol{M}}_{k}=\mathbf{E}\left[\mathbf{M}_{k}\right]=$

$\left\{(\widehat{\mathbf{W}} \widehat{\mathbf{H}})_{n p} \mid(n, p) \in \mathcal{S}_{k}\right\}$, for the $k^{t h}$ fold, $k=1,2, \ldots, K$

$$
E R R_{r, k} \equiv-\log \left\{\operatorname{Pr}\left(\mathbf{V}_{k}^{L} \mid \widehat{\boldsymbol{M}}_{k}\right)\right\}=D_{K L}\left(\mathbf{V}_{k}^{L} \mid \widehat{\boldsymbol{M}}_{k}\right)-C^{*}
$$

We then evaluate overall prediction error, $E R R_{r}=\sum_{k=1}^{K} E R R_{r, k}$, across $\mathrm{K}$ folds. Since the term $C^{*}$ is unrelated to $\widehat{\mathbf{W}}$ and $\widehat{\mathbf{H}}$, it is dropped. Finally, we select the number of signatures $r^{*}$ which minimizes $E R R_{r}$ over a range of numbers of signatures $1 \leq r \leq R$.

\section{Extracting signature profiles and estimating contributions of signatures}

Once the optimal number of signatures $r^{*}$ is determined by SUITOR, we extract mutational signature profiles $\mathbf{W}$ and estimating contributions $\mathbf{H}$, via maximizing $\log \{\operatorname{Pr}(\mathbf{V} \mid \mathbf{W H})\}$ with the fixed rank $r^{*}$. Similar to the ECM algorithm in SUITOR, we evaluate multiple initial values and use $\widehat{\mathbf{W}}$ and $\widehat{\mathbf{H}}$ which maximizes $\log \{\operatorname{Pr}(\mathbf{V} \mid \widehat{\mathbf{W}} \widehat{\mathbf{H}})\}$ to relieve local optima problem.

\section{In vitro studies}

The datasets of two in vitro studies were downloaded from

http://medgen.medschl.cam.ac.uk/serena-nik-zainal/. The details of study design and implementation were described previously ${ }^{2,5}$. In these in vitro studies, the endogenous and exogenous mutational signatures were experimentally generated in vitro and hence the true number of signatures and profiles are known.

We create the mutation catalog matrix for both studies and apply SUITOR, sigProfiler, signatureAnalyzer and signeR. We choose sigProfiler and signatureAnalyzer (with the default 
setting of prior), since they have been applied to a number of studies ${ }^{7,8,18,39-41}$, and signe ${ }^{17}$ because it utilizes Bayesian information criterion (BIC), a popular model selection criterial for supervised learning. For SUITOR, we use 10-fold cross-validation with $90 \%$ of counts in mutation catalog matrix as the training set and the remaining $10 \%$ as the validation set. In contrast, the whole mutation catalog matrix $\mathbf{V}$ is analyzed by sigProfiler, signatureAnalyzer and signeR respectively under the default setting.

The first study induces endogenous mutational signatures by CRISPR-Cas9-mediated knockouts of DNA repair genes in an isogenic human cell line. The mutations of each cell line were called based on Whole-genome sequencing (WGS). First, we focus on the MSH6 knockout-induced single base substitution signature, which is characterized by $\mathrm{C}>\mathrm{T}$ and $\mathrm{T}>\mathrm{C}$ single base substitutions ( 148 substitutions per cell division). We evaluate if the four methods are able to retrieve the background signature and the $M S H 6$ knockout-induced signatures. Next, we analyze the gene-knockout studies with no induced signatures (for genes CHK2, NEIL1, NUDT1, POLB, POLE and POLM), to evaluate if the four methods would find false positive signatures in addition to the background signature.

In the second study, exogenous mutational signatures were created by environmental or therapeutic mutagens. We select 35 controls and 52 subclones of human-induced pluripotent stem cell (iPSC) lines, for which the mutations were called based on whole-genome sequencing (WGS). While controls are not exposed to mutagens, each subclone is exposed to one of eight mutagens, including simulated solar radiation (SSR), dibenzo[a,1]pyrene (DBP) and alkylating agent therapy temozolomide (TMZ). SSR recapitulates the UV-associated signatures and DBP is 
a potent carcinogen of the polycyclic aromatic hydrocarbons (PAHs) produced when coal, crude oil, or gasoline is burned. For each method, we check if the correct number of signatures will be attained with its impacts on the downstream analyses. Specifically, we investigate if the retrieved de novo signature profiles are highly similar to the true signature profiles. We further explore if signature contributes could separate subclones exposed to the distinctive mutagens, visualized by $\mathrm{t}$-distributed stochastic neighbor embedding $\left(\mathrm{t}-\mathrm{SNE}^{42}\right)$.

\section{In silico simulation design with one signature}

We simulated a mutation catalog matrix for 500 tumors and analyzed it by the four methods, each repeated 20 times. we used the signature profile of SBS8 as the true signature profile for $\mathbf{H}$ and generated the contribution vector $\mathbf{W}$ from a uniform distribution within the range [20000, 40000]. Then the mutation catalog $\mathbf{V}$ is generated by a Poisson distribution with mean $\mathbf{W H}$.

\section{In silico simulation design with nine signatures}

We simulated signatures mimic to the ones observed in the Pan-Cancer Analysis of Whole Genomes (PCAWG) breast cancer study ${ }^{1}$. The nine signatures show various signature contributions and signature profiles; some signatures contribute to all tumors (e.g., SBS1 and SBS5, present in $100 \%$ of tumors; Supplementary Table 3) while others contribute to a few tumors (e.g., SBS41, present in $6 \%$ of tumors) and even to one (SBS40) or two tumors (SBS8); some signature profiles are spiky (e.g., SBS1 and SBS2/13; Supplementary Fig. 5) while others are relatively flat (e.g., SBS5). 
Specifically, we generated mutation catalog matrices similar to $\mathbf{V}^{\mathrm{BR}}$, the mutation catalog matrix of PCAWG breast cancer study. $\mathbf{V}^{\mathrm{BR}}$ is approximated by $\mathbf{W}^{\mathrm{BR}} \mathbf{H}$, for which $\mathbf{H}$ contains the COSMIC signature profiles and $\mathbf{W}^{\mathrm{BR}}$ is the corresponding signature contribution matrix (downloaded from https://www.synapse.org/\#!Synapse:syn11738669). We removed signatures with zero contributions to all tumors and chose 9 signatures to compose the signature profile matrix $\mathbf{H}$ of size $9 \times 96$. With $\mathbf{H}$ fixed, we took bootstrap samples $\mathbf{W}_{\boldsymbol{b o o t}}^{\boldsymbol{B R}}$ from each column of matrix $\mathbf{W}^{\mathrm{BR}}$, and generated $\mathbf{V}_{\text {boot }}^{\boldsymbol{B R}}$ which follows a Poisson distribution with mean $\mathbf{W}_{\boldsymbol{b o o t}}^{\boldsymbol{B R}} \mathbf{H}$. Due to the dependencies between SBS1 and SBS5 as well as between SBS2 and SBS13, their contributions are resampled jointly while the contributions of other signatures are sampled individually. We simulated 20 mutation catalog matrices for 200 tumors and each was analyzed by four methods respectively.

\section{In vivo human cancer genomics studies}

We analyzed 1,539 tumors of eight cancer types with at least 100 tumors each from $\mathrm{PCAWG}^{1}$ : 321 tumors of hepatocellular carcinoma, 286 tumors of prostate adenocarcinoma, 237 tumors of pancreatic adenocarcinoma, 194 tumors of breast adenocarcinoma, 146 tumors of central nervous system medulloblastoma, 143 tumors of renal cell carcinoma,112 tumors of ovary adenocarcinoma and 100 cases of B-cell non-Hodgkin lymphoma. The tumors were whole genome sequenced and datasets of the somatic mutation calls were downloaded from https://www.synapse.org/\#!Synapse:syn11726620. The hypermutator tumors with mutation burden more than 10 mutations/Mb were excluded ${ }^{43}$. For each cancer type, we applied SUITOR, sigProfiler, signatureAnalyzer and signeR to select the number of signatures and estimate signature contributions and profiles. 
To compare the prediction errors, we split the mutation catalog matrix into a training set (90\% of counts in the mutation catalog matrix), a validation set (5\%) and a testing set (5\%). For SUITOR, the training set was used to fit the probabilistic NMF model with multiple numbers of signatures and the validation set to select the number of signatures. The other methods used both the training and validation sets to select the number of signatures. Next, we compared the prediction errors of selected signatures by each method on the testing set. For sigProfiler, signatureAnalyzer and signeR, we imputed missing training data by medians of available mutation counts per each mutational catalog, applied each method, predicted the testing data and calculated the prediction error as SUITOR did. For SUITOR, it could handle missing data and predict the testing data by the ECM algorithm.

\section{Sanger whole genome sequencing breast cancer study}

Sanger whole genome breast cancer study sequenced 560 breast tumors. The somatic mutation calls files were downloaded from https://medgen.medschl.cam.ac.uk/serena-nik-zainal/. Among 560 breast tumors, 110 tumors are included in PCAWG and hence excluded from this validation study. Ten hypermutator tumors are also excluded. We apply SUITOR, sigProfiler, signatureAnalyzer and signeR to a) select the number of signatures and estimate signature contributions and profiles; b) compare the signatures with ones detected in the PCAWG breast cancer study; and c) investigate if additional signatures are found in Sanger whole genome breast cancer study. In addition, we stratify the tumors based on mutation contributions and associate the signature clusters with epidemiological or clinical characteristics. 


\section{Software webpages:}

SUITOR: https://github.com/binzhulab/SUITOR

sigProfiler: v1.0.6 is installed and used by following instructions in https://github.com/AlexandrovLab/SigProfilerExtractor.

signatureAnalyzer: version information is not available but is installed by following instructions in https://github.com/broadinstitute/getzlab-SignatureAnalyzer.

signeR: v1.12.0 is downloaded from https://bioconductor.org/packages/release/bioc/html/signeR.html.

sigProfiler and signatureAnalyzer are used in python version 3.7.5 while SUITOR and signeR are used in $\mathrm{R}$ version 3.6.3. Signature profiles plot is drawn based on $\mathrm{R}$ package MutationalPatterns from https://bioconductor.org/packages/release/bioc/html/MutationalPatterns.html with slight modification to add graphical parameters.

\section{Data availability}

All datasets are publicly available. The datasets of in vitro studies and Sanger whole genome breast cancer study: https://medgen.medschl.cam.ac.uk/serena-nik-zainal/; the datasets of PanCancer Analysis of Whole Genomes (PCAWG) breast cancer study:

https://www.synapse.org/\#!Synapse:syn11726620 


\section{Acknowledgment}

This research was supported by the Intramural Research Program of the National Institutes of

Health, National Cancer Institute, Division of Cancer Epidemiology and Genetics (DCEG). This study utilized the high-performance computational capabilities of the Biowulf Linux cluster at the National Institutes of Health, Bethesda, MD: https://biowulf.nih.gov. We would like to thank Bill Wheeler (Information Management Services) for computation support and Dr. Paul Albert (Biostatistics Branch of DCEG) and Dr. Ludmil Alexandrov (University of California San Diego) for helpful comments. Conflict of Interest: None declared.

\section{Reference:}

1. Alexandrov, L.B. et al. The repertoire of mutational signatures in human cancer. Nature 578, 94-101 (2020).

2. Kucab, J.E. et al. A Compendium of Mutational Signatures of Environmental Agents. Cell 177, 821-836 e16 (2019).

3. Alexandrov, L.B. et al. Mutational signatures associated with tobacco smoking in human cancer. Science 354, 618-622 (2016).

4. Letouze, E. et al. Mutational signatures reveal the dynamic interplay of risk factors and cellular processes during liver tumorigenesis. Nat Commun 8, 1315 (2017).

5. Zou, X. et al. Validating the concept of mutational signatures with isogenic cell models. Nat Commun 9, 1744 (2018).

6. Polak, P. et al. A mutational signature reveals alterations underlying deficient homologous recombination repair in breast cancer. Nat Genet 49, 1476-1486 (2017).

7. Haradhvala, N.J. et al. Distinct mutational signatures characterize concurrent loss of polymerase proofreading and mismatch repair. Nat Commun 9, 1746 (2018).

8. Petljak, M. et al. Characterizing Mutational Signatures in Human Cancer Cell Lines Reveals Episodic APOBEC Mutagenesis. Cell 176, 1282-1294 e20 (2019).

9. Drost, J. et al. Use of CRISPR-modified human stem cell organoids to study the origin of mutational signatures in cancer. Science 358, 234-238 (2017).

10. Secrier, M. et al. Mutational signatures in esophageal adenocarcinoma define etiologically distinct subgroups with therapeutic relevance. Nat Genet 48, 1131-41 (2016).

11. Staaf, J. et al. Whole-genome sequencing of triple-negative breast cancers in a population-based clinical study. Nat Med 25, 1526-1533 (2019).

12. Connor, A.A. et al. Association of Distinct Mutational Signatures With Correlates of Increased Immune Activity in Pancreatic Ductal Adenocarcinoma. JAMA Oncol 3, 774783 (2017). 
13. Li, X. et al. Distinct Subtypes of Gastric Cancer Defined by Molecular Characterization Include Novel Mutational Signatures with Prognostic Capability. Cancer Res 76, 1724-32 (2016).

14. Gehring, J.S., Fischer, B., Lawrence, M. \& Huber, W. SomaticSignatures: inferring mutational signatures from single-nucleotide variants. Bioinformatics 31, 3673-5 (2015).

15. Alexandrov, L.B., Nik-Zainal, S., Wedge, D.C., Campbell, P.J. \& Stratton, M.R. Deciphering signatures of mutational processes operative in human cancer. Cell Rep 3 , 246-59 (2013).

16. Fischer, A., Illingworth, C.J., Campbell, P.J. \& Mustonen, V. EMu: probabilistic inference of mutational processes and their localization in the cancer genome. Genome Biol 14, R39 (2013).

17. Rosales, R.A., Drummond, R.D., Valieris, R., Dias-Neto, E. \& da Silva, I.T. signeR: an empirical Bayesian approach to mutational signature discovery. Bioinformatics 33, 8-16 (2017).

18. Kim, J. et al. Somatic ERCC2 mutations are associated with a distinct genomic signature in urothelial tumors. Nat Genet 48, 600-606 (2016).

19. Rosenthal, R., McGranahan, N., Herrero, J., Taylor, B.S. \& Swanton, C. DeconstructSigs: delineating mutational processes in single tumors distinguishes DNA repair deficiencies and patterns of carcinoma evolution. Genome Biol 17, 31 (2016).

20. Huang, X., Wojtowicz, D. \& Przytycka, T.M. Detecting presence of mutational signatures in cancer with confidence. Bioinformatics 34, 330-337 (2018).

21. Maura, F. et al. A practical guide for mutational signature analysis in hematological malignancies. Nat Commun 10, 2969 (2019).

22. Degasperi, A. et al. A practical framework and online tool for mutational signature analyses show inter-tissue variation and driver dependencies. Nat Cancer 1, 249-263 (2020).

23. Schwarz, G. Estimating the dimension of a model. The annals of statistics 6, 461-464 (1978).

24. Tan, V.Y. \& Févotte, C. Automatic relevance determination in nonnegative matrix factorization with the $\beta$-divergence. IEEE Trans Pattern Anal Mach Intell 35, 1592-605 (2013).

25. Arlot, S. \& Celisse, A. A survey of cross-validation procedures for model selection. Statistics surveys 4, 40-79 (2010).

26. Meng, X.-L. \& Rubin, D.B. Maximum Likelihood Estimation via the ECM Algorithm: A General Framework. Biometrika 80, 267-278 (1993).

27. Févotte, C. \& Cemgil, A.T. Nonnegative matrix factorizations as probabilistic inference in composite models. in 2009 17th European Signal Processing Conference 1913-1917 (2009).

28. Gaussier, E. \& Goutte, C. Relation between PLSA and NMF and implications. in Proceedings of the 28th annual international ACM SIGIR conference on Research and development in information retrieval 601-602 (Association for Computing Machinery, Salvador, Brazil, 2005).

29. Friedman, J., Hastie, T. \& Tibshirani, R. The elements of statistical learning, (Springer series in statistics New York, 2001).

30. Pavlidis, N. \& Fizazi, K. Carcinoma of unknown primary (CUP). Crit Rev Oncol Hematol 69, 271-8 (2009).

31. Nik-Zainal, S. et al. Landscape of somatic mutations in 560 breast cancer whole-genome sequences. Nature 534, 47-54 (2016).

32. Little, R.J. \& Rubin, D.B. Statistical analysis with missing data, (John Wiley \& Sons, 2019). 
33. Kohavi, R. A study of cross-validation and bootstrap for accuracy estimation and model selection. in Proceedings of the 14th international joint conference on Artificial intelligence - Volume 21137-1143 (Morgan Kaufmann Publishers Inc., Montreal, Quebec, Canada, 1995).

34. Breiman, L. \& Spector, P. Submodel Selection and Evaluation in Regression. The XRandom Case. International Statistical Review / Revue Internationale de Statistique 60, 291-319 (1992).

35. Ding, C., Li, T. \& Peng, W. Nonnegative matrix factorization and probabilistic latent semantic indexing: equivalence, chi-square statistic, and a hybrid method. in Proceedings of the 21st national conference on Artificial intelligence - Volume 1342-347 (AAAI Press, Boston, Massachusetts, 2006).

36. Lee, D.D. \& Seung, H.S. Learning the parts of objects by non-negative matrix factorization. Nature 401, 788-91 (1999).

37. Bro, R., Kjeldahl, K., Smilde, A.K. \& Kiers, H.A.L. Cross-validation of component models: A critical look at current methods. Analytical and Bioanalytical Chemistry 390, 1241-1251 (2008).

38. Wold, S. Cross-Validatory Estimation of the Number of Components in Factor and Principal Components Models. Technometrics 20, 397-405 (1978).

39. Xue, R. et al. Genomic and Transcriptomic Profiling of Combined Hepatocellular and Intrahepatic Cholangiocarcinoma Reveals Distinct Molecular Subtypes. Cancer Cell 35, 932-947 e8 (2019).

40. Rose Li, Y. et al. Mutational signatures in tumours induced by high and low energy radiation in Trp53 deficient mice. Nat Commun 11, 394 (2020).

41. Kasar, S. et al. Whole-genome sequencing reveals activation-induced cytidine deaminase signatures during indolent chronic lymphocytic leukaemia evolution. Nat Commun 6, 8866 (2015).

42. Maaten, L.v.d. \& Hinton, G. Visualizing data using t-SNE. Journal of machine learning research 9, 2579-2605 (2008).

43. Campbell, B.B. et al. Comprehensive Analysis of Hypermutation in Human Cancer. Cell 171, 1042-1056 e10 (2017).

\section{Figure legends:}

Fig.1 A schematic overview of SUITOR. This schematic diagram illustrates how SUITOR selects

the number of de novo mutational signatures. Details are given in Results. Each row of the mutation catalog matrix represents a tumor, each column a mutation catalog. The prediction error curves in the training set (blue) and validation set (red) are manually drawn for the illustration purpose, with the red dot representing the minimal prediction error in the validation set. ECM algorithm: expectation/conditional maximization algorithm; CM steps: conditional maximization steps; E steps: expectation steps. 
Fig.2 In vitro evaluation of SUITOR and other methods. a) Prediction errors of SUITOR for the training and validation sets of in vitro knockout study of DNA repair gene MSH6. The red dot denotes the number of signatures with the minimal prediction error in the validation set. b) Profiles of single base substitution signature estimated by SUITOR in MSH6 gene knockout study. The $x$-axis indicates the $5^{\prime}$ and $3^{\prime}$ nucleotides for each substitution type (e.g., C>A). The top panel: the true and estimated background signatures (cosine similarity=0.991; cosine similarity=1 suggests two profiles being identical.); the bottom panel: the true and estimated MSH6 knockout-induced signatures (cosine similarity=0.997). c) Prediction errors by SUITOR for the training and validation sets of in vitro study of eight environmental mutagens. d) Clusters of subclones visualized by t-SNE for SUITOR, sigProfiler, signatureAnalyzer and signeR. Each dot represents a subclone, colored by the mutagen treatment. 1,8-DNP: 1,8-Dinitropyrene; PhIP:2amino-1-methyl-6-phenylimidazo[4,5-b]pyridine; 6-NC: 6-Nitrochrysene; DBA: Dibenz[a,h]anthracene; DBP: Dibenzo[a,I]pyrene; KBrO3: Potassium bromate; SSR: Simulated solar radiation; TMZ: Temozolomide.

Fig.3 In silico evaluation of SUITOR and other methods. a) The signature profiles of the true mutational signature and the ones discovered by each method for a replicate. b) The number of replicates in which a given signature will be detected by each method; the rare signatures SBS8 and SBS41 were discovered in 12, 6, 14 and 6 replicates, and 17, 7, 19 and 11 replicates over total 20 replicates for SUITOR, sigProfiler, signatureAnalyzer and signR, respectively. 
Fig.4 Mutational signature results of eight cancer studies of PCAWG. a) The prediction errors (in the log10 scale) of SUITOR, sigProfiler, signatureAnalyzer and signeR for eight cancer types and for all tumors together, namely PCAWG8. SD: standard deviation. b) The number of signatures identified by each method. The shaded bars and the numbers above indicate the number of signatures whose profiles could be matched to COSMIC profiles (with cosine similarity $>0.8)$. c) The cosine similarities between de novo signatures and COSMIC signatures. Only the matched pairs are shown (with cosine similarity $>0.8$ ). The higher the cosine similarity indicates the better match to a COSMIC signature profile. The cosine similarity equivalent to one means the perfect match.

Fig.5 The t-SNE visualization of clustering patterns based on signature contributions. Signature contributions are estimated by four methods: (a) SUITOR, (b) sigProfiler, (c) signatureAnalyzer and (d) signeR. Each dot represents a tumor and is colored by the cancer type.

Fig.6 The results of Sanger breast cancer study by SUITOR. a) The heatmap of cosine similarity between de novo signatures detected in PCAWG breast cancer (BRCA) study and de novo signatures in Sanger BRCA study. The signatures of Sanger BRCA study are annotated by COSMIC signatures (if cosine similarities $>0.8$ ) in the parentheses. For example, DNSigA (SBS1) refers to de novo signature A (DNSigA) being annotated by COSMIC signature SBS1. b) The heatmap of signature contributions estimated by SUITOR with hierarchical clustering in Sanger BRCA study. The number of tumors included in each signature cluster is shown in the 
parentheses on the top of the heatmap. Q1, Q2, Q3: the 1st, 2nd and 3rd quantiles of signature contributions. c) The t-SNE visualization of tumors in G3 and G4 signature clusters, color-coded by signature clusters and molecular subtypes. TNBC: triple negative breast cancer. 


\section{Figures}

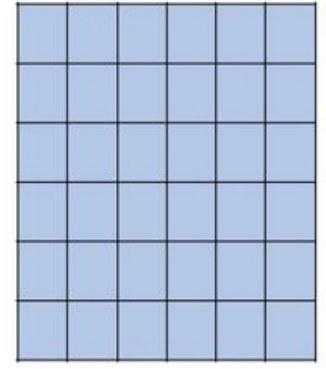

Mutation catalog matrix

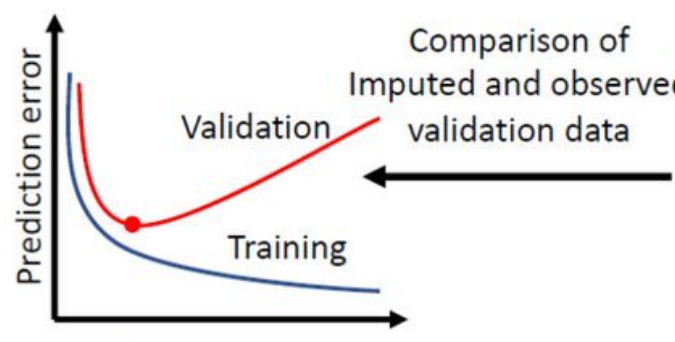

Number of signatures

Create the training set: blank cells present missing data reserved as validation data

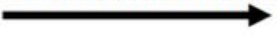

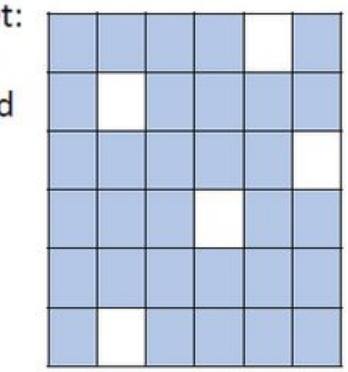
make some guess

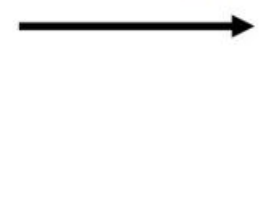

CM steps: estimate $\mathbf{W}$ and $\mathbf{H}$

Final step: impute missing validation data
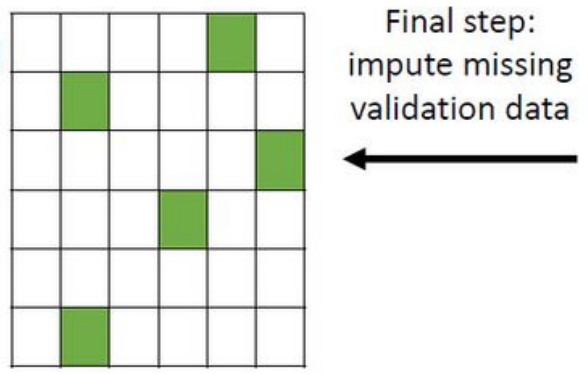

E step: update missing values

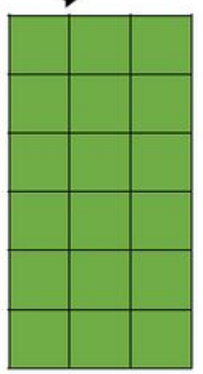

\section{Figure 1}

A schematic overview of SUITOR. This schematic diagram illustrates how SUITOR selects the number of de novo mutational signatures. Details are given in Results. Each row of the mutation catalog matrix represents a tumor, each column a mutation catalog. The prediction error curves in the training set (blue) and validation set (red) are manually drawn for the illustration purpose, with the red dot representing the minimal prediction error in the validation set. ECM algorithm: expectation/conditional maximization algorithm; CM steps: conditional maximization steps; E steps: expectation steps. 


\section{a}

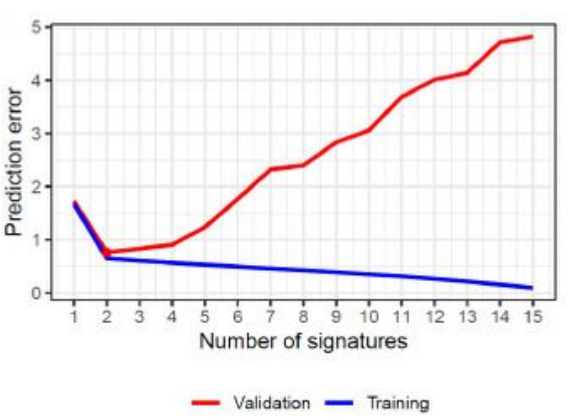

$\mathrm{C}$

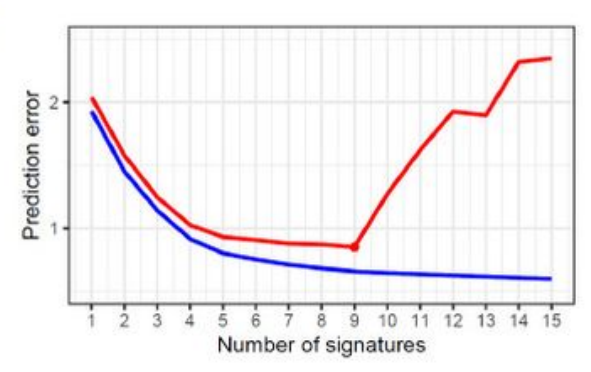

- Validation - Training
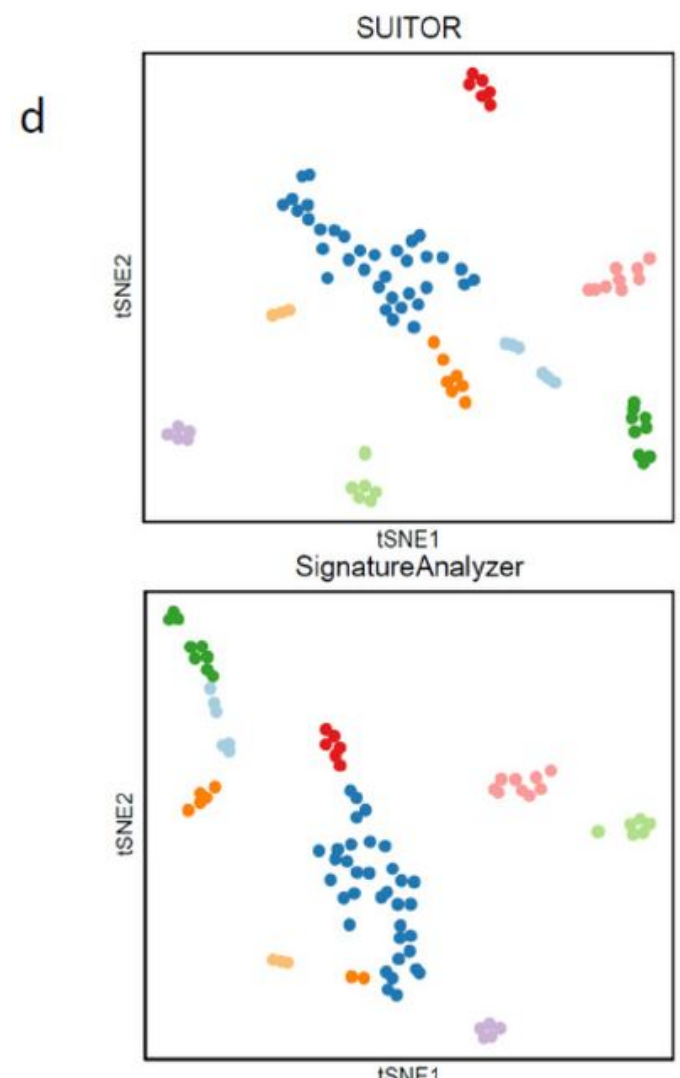

b

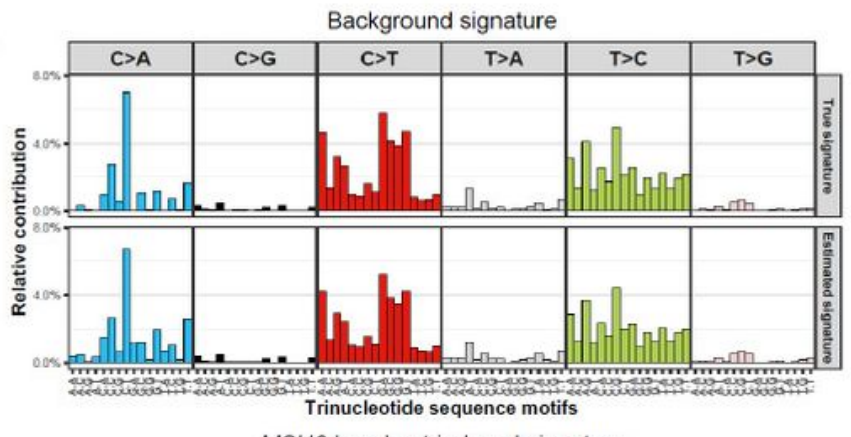

MSH6 knockout-induced signature
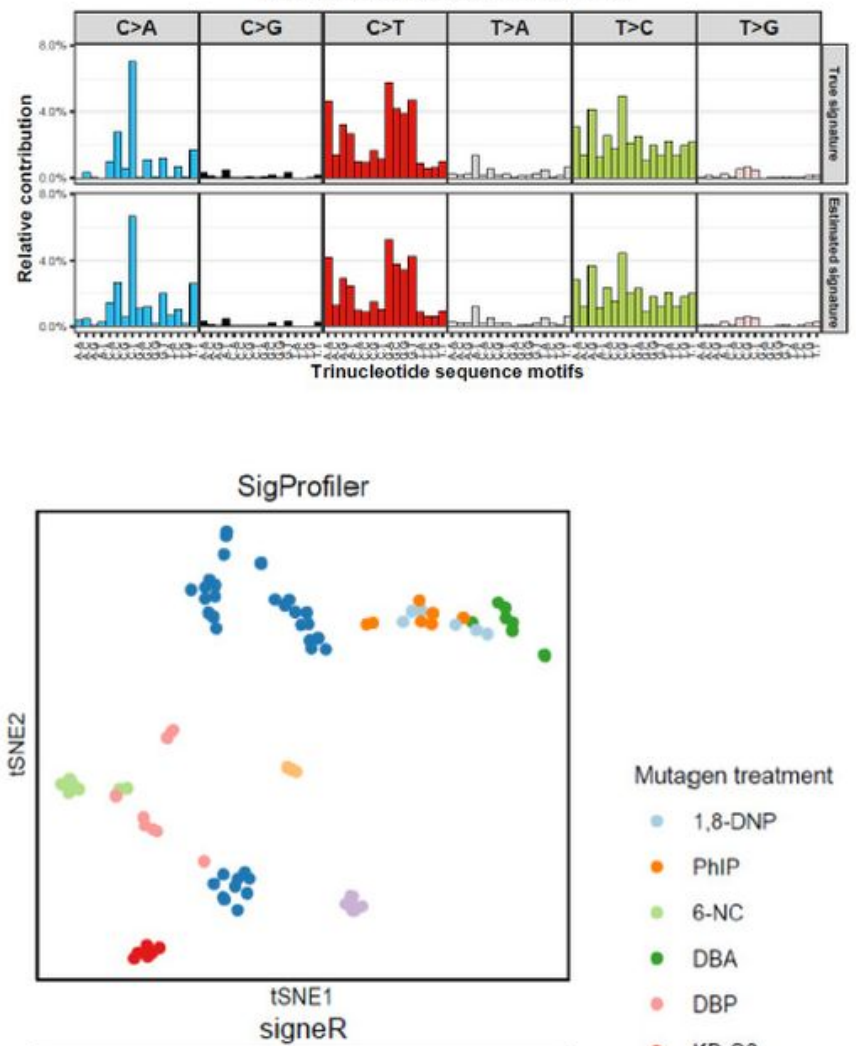

Mutagen treatment

- 1.8-DNP

- PhIP

- 6-NC

- DBA

- DBP

- $\mathrm{KBrO} 3$

- SSR

- TMZ

- Absence

\section{Figure 2}

In vitro evaluation of SUITOR and other methods. a) Prediction errors of SUITOR for the training and validation sets of in vitro knockout study of DNA repair gene MSH6. The red dot denotes the number of signatures with the minimal prediction error in the validation set. b) Profiles of single base substitution signature estimated by SUITOR in MSH6 gene knockout study. The x-axis indicates the $5^{\prime}$ and 3' nucleotides for each substitution type (e.g., C>A). The top panel: the true and estimated background 
signatures (cosine similarity $=0.991$; cosine similarity $=1$ suggests two profiles being identical.); the bottom panel: the true and estimated MSH6 knockout-induced signatures (cosine similarity=0.997). c) Prediction errors by SUITOR for the training and validation sets of in vitro study of eight environmental mutagens. d) Clusters of subclones visualized by t-SNE for SUITOR, sigProfiler, signatureAnalyzer and signeR. Each dot represents a subclone, colored by the mutagen treatment. 1,8-DNP: 1,8-Dinitropyrene; PhIP:2-amino-1methyl-6-phenylimidazo[4,5-b]pyridine; 6-NC: 6-Nitrochrysene; DBA: Dibenz[a,h]anthracene; DBP: Dibenzo[a,I]pyrene; KBrO3: Potassium bromate; SSR: Simulated solar radiation; TMZ: Temozolomide.

\section{a}
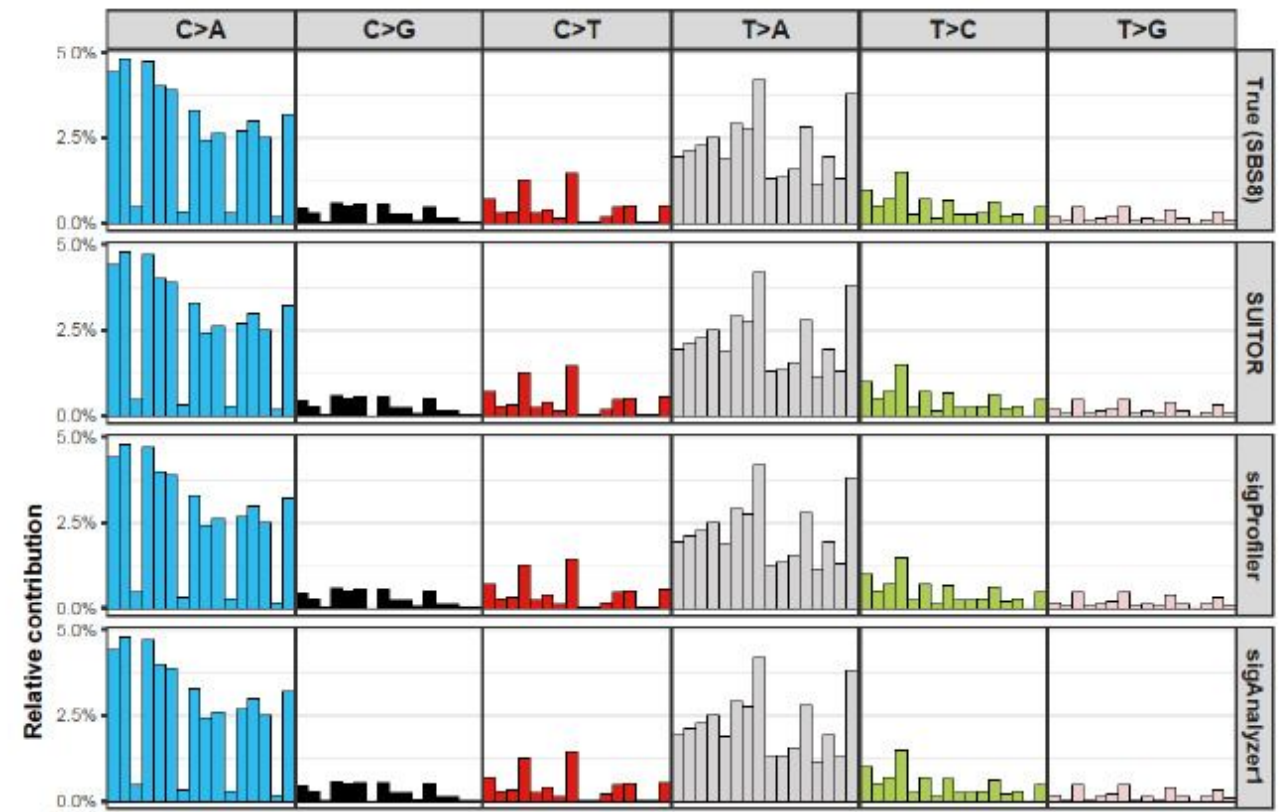

b
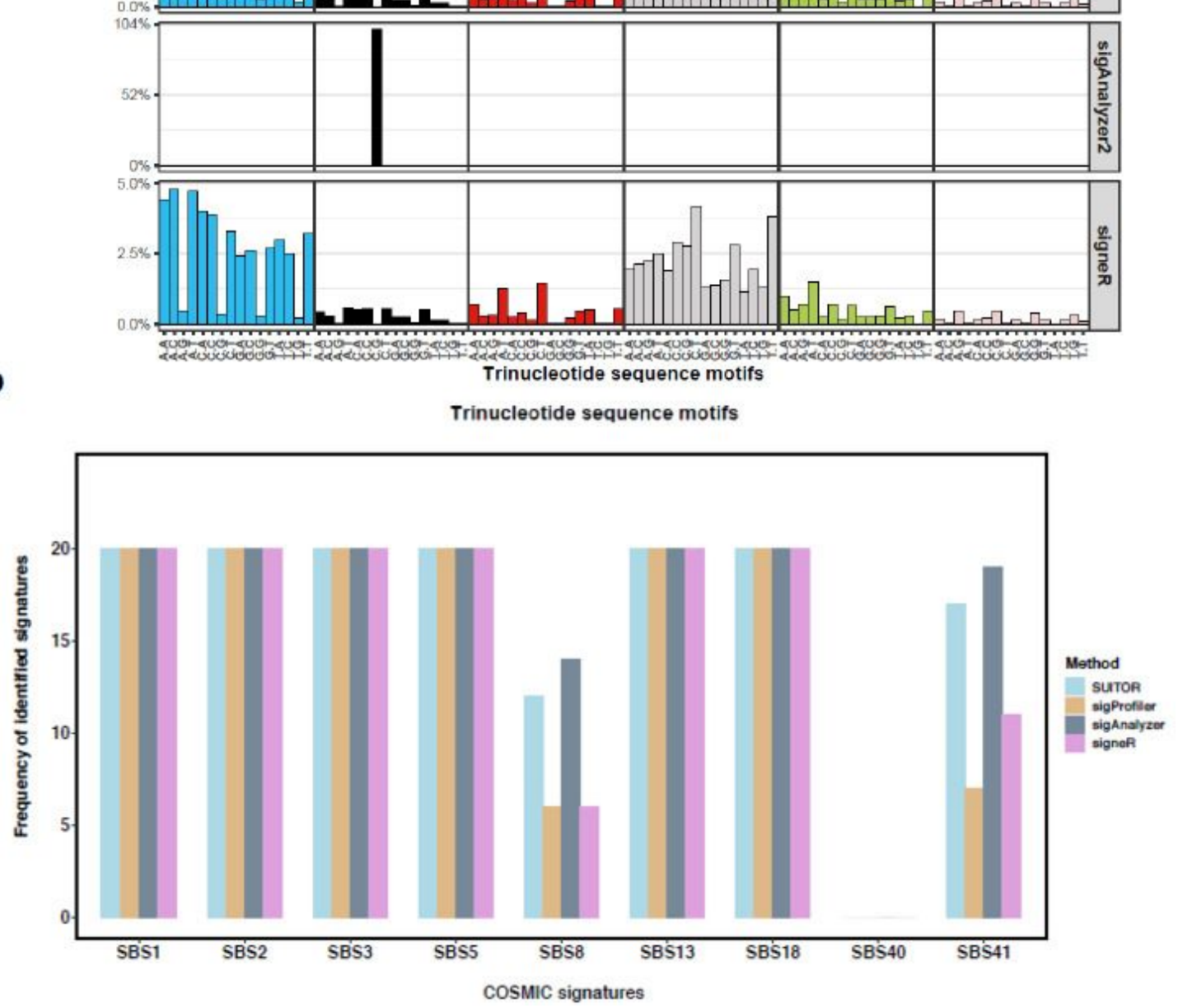


\section{Figure 3}

In silico evaluation of SUITOR and other methods. a) The signature profiles of the true mutational signature and the ones discovered by each method for a replicate. b) The number of replicates in which a given signature will be detected by each method; the rare signatures SBS8 and SBS41 were discovered in 12, 6, 14 and 6 replicates, and 17, 7, 19 and 11 replicates over total 20 replicates for SUITOR, sigProfiler, signatureAnalyzer and signR, respectively.
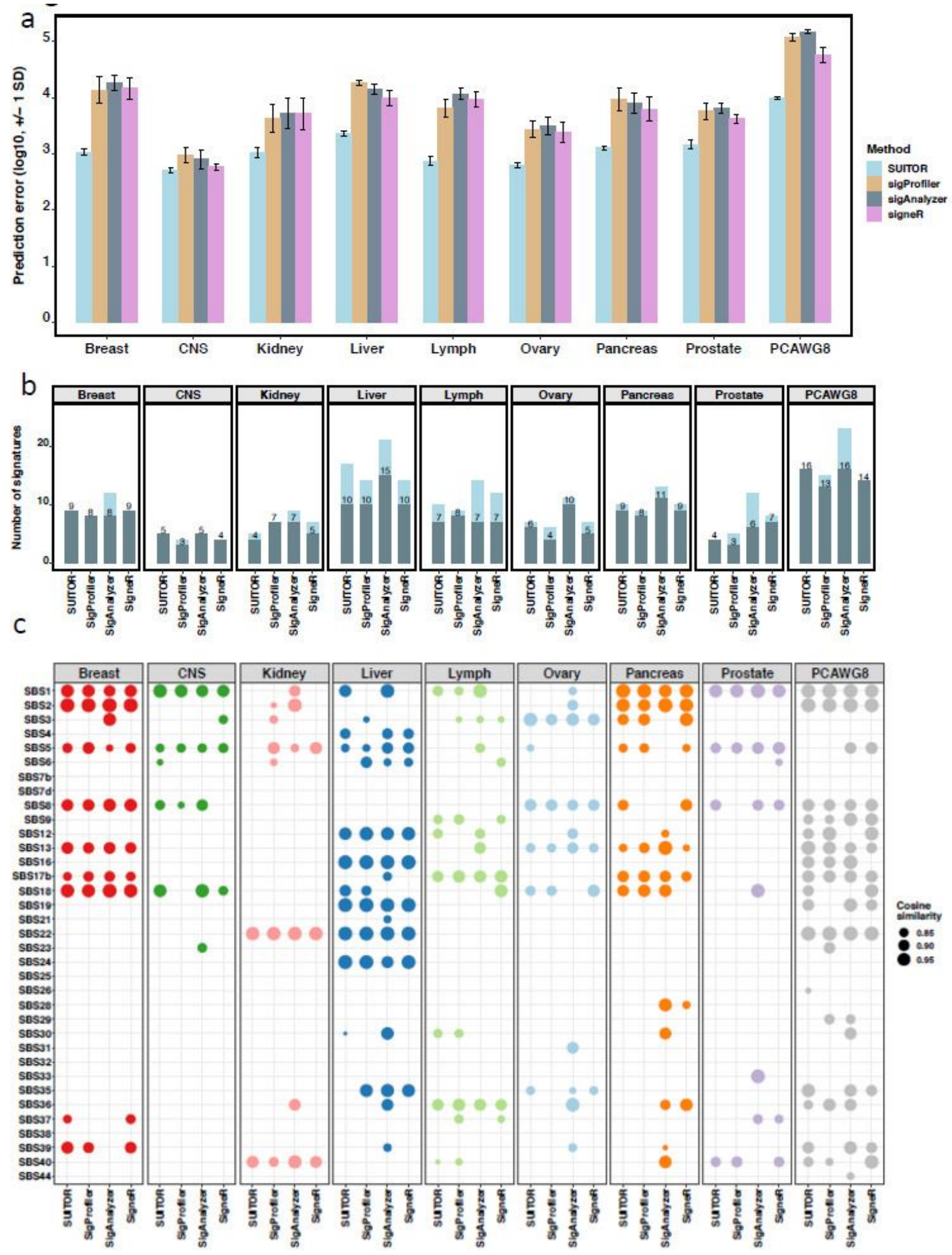


\section{Figure 4}

Mutational signature results of eight cancer studies of PCAWG. a) The prediction errors (in the log10 scale) of SUITOR, sigProfiler, signatureAnalyzer and signeR for eight cancer types and for all tumors together, namely PCAWG8. SD: standard deviation. b) The number of signatures identified by each method. The shaded bars and the numbers above indicate the number of signatures whose profiles could be matched to COSMIC profiles (with cosine similarity $>0.8$ ). c) The cosine similarities between de novo signatures and COSMIC signatures. Only the matched pairs are shown (with cosine similarity $>0.8$ ). The higher the cosine similarity indicates the better match to a COSMIC signature profile. The cosine similarity equivalent to one means the perfect match. 


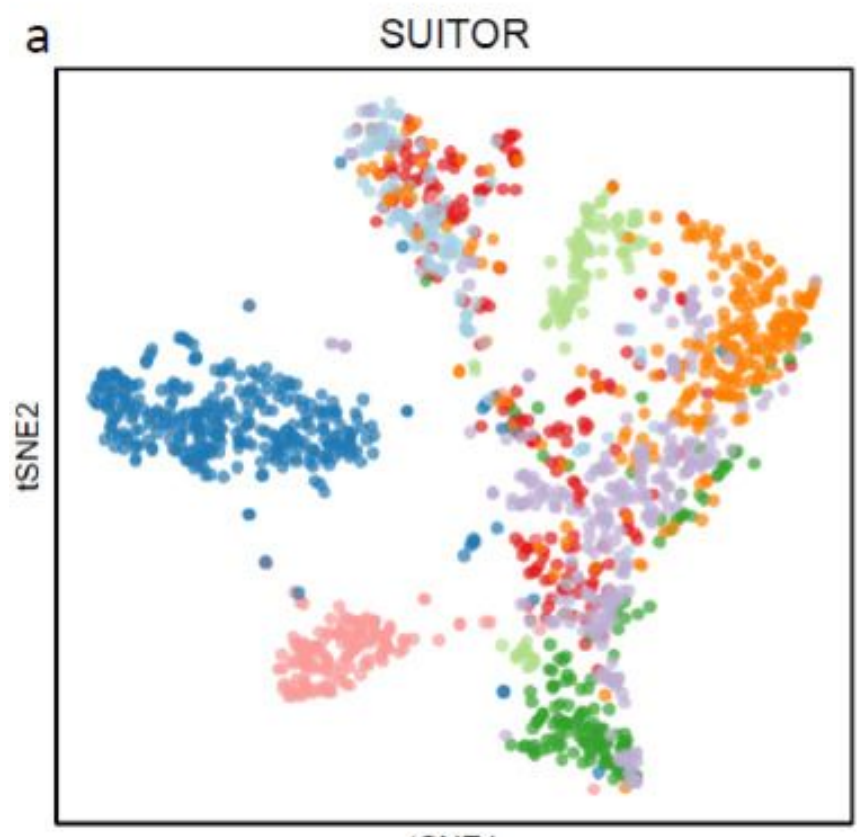

tSNE 1

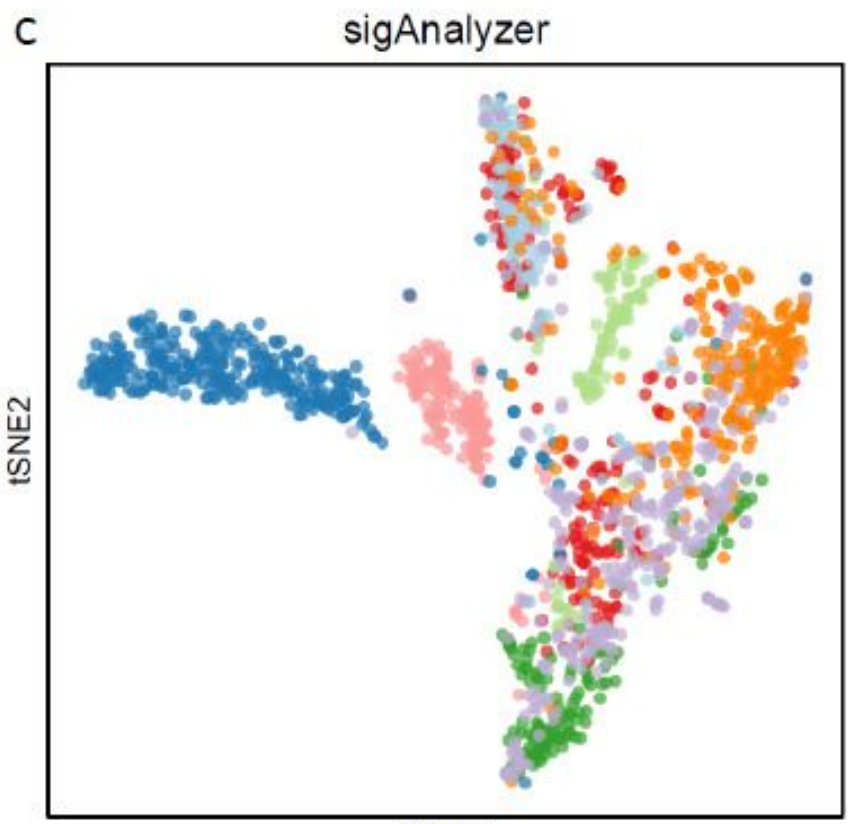

tSNE1

Cancer type

-

Breast CNS b sigProfiler

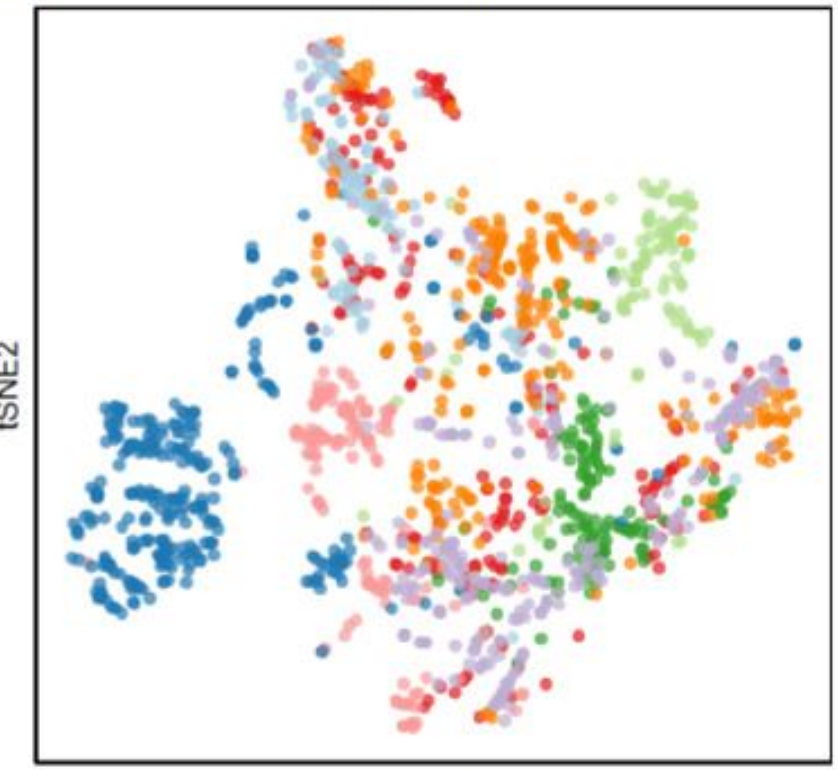

tSNE1

d

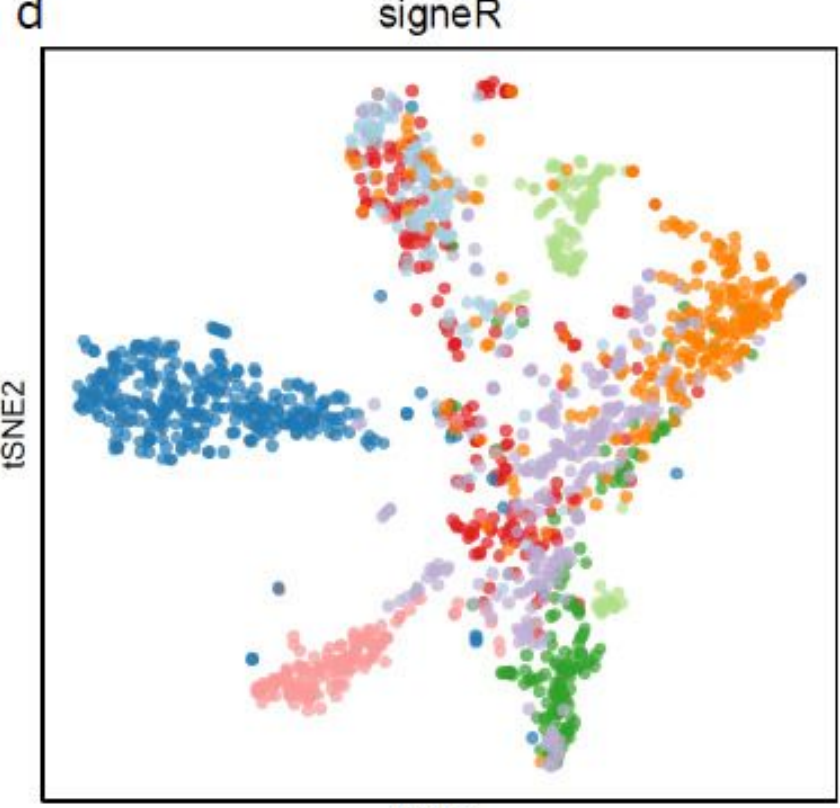

tSNE1

Lymph Ovary Pancreas

Liver

Prostate

\section{Figure 5}

The t-SNE visualization of clustering patterns based on signature contributions. Signature contributions are estimated by four methods: (a) SUITOR, (b) sigProfiler, (c) signatureAnalyzer and (d) signeR. Each dot represents a tumor and is colored by the cancer type. 
a

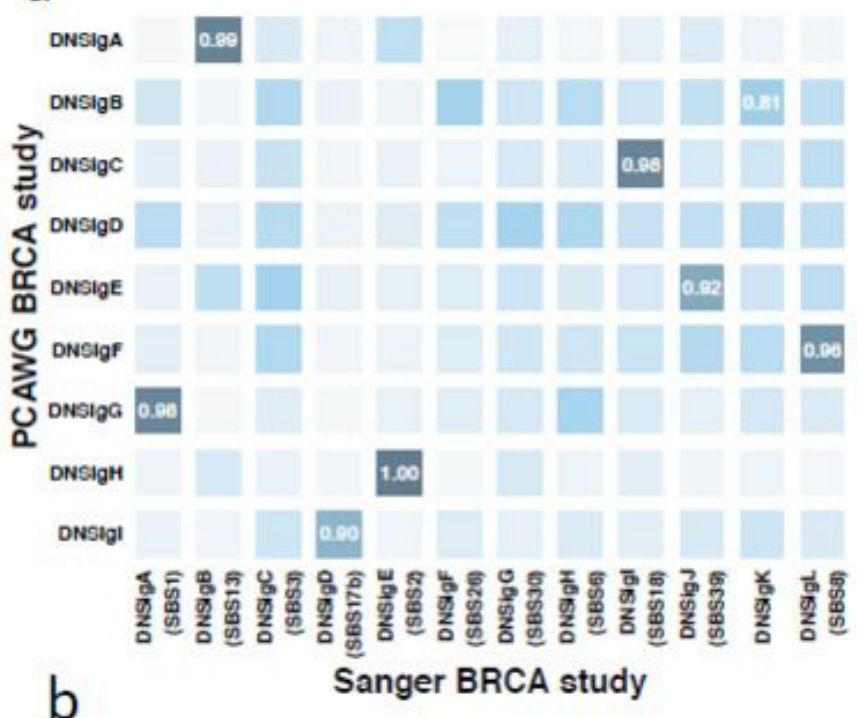

b
C

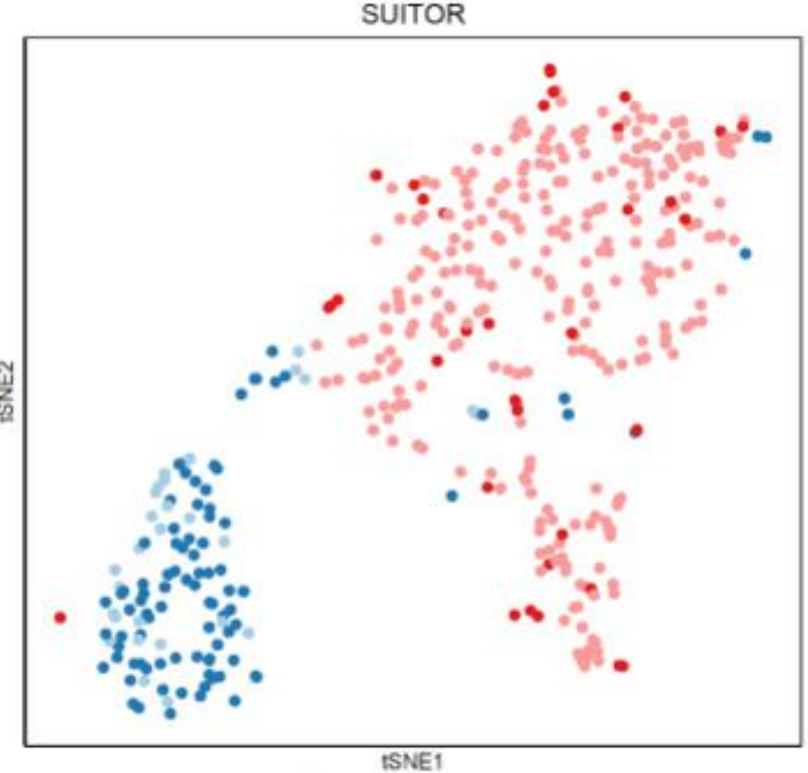

tSNE1

Subtypes - G3/TNBC * G3/OtherSubtypes
- G4TNBC * G4/OtherSubtypes

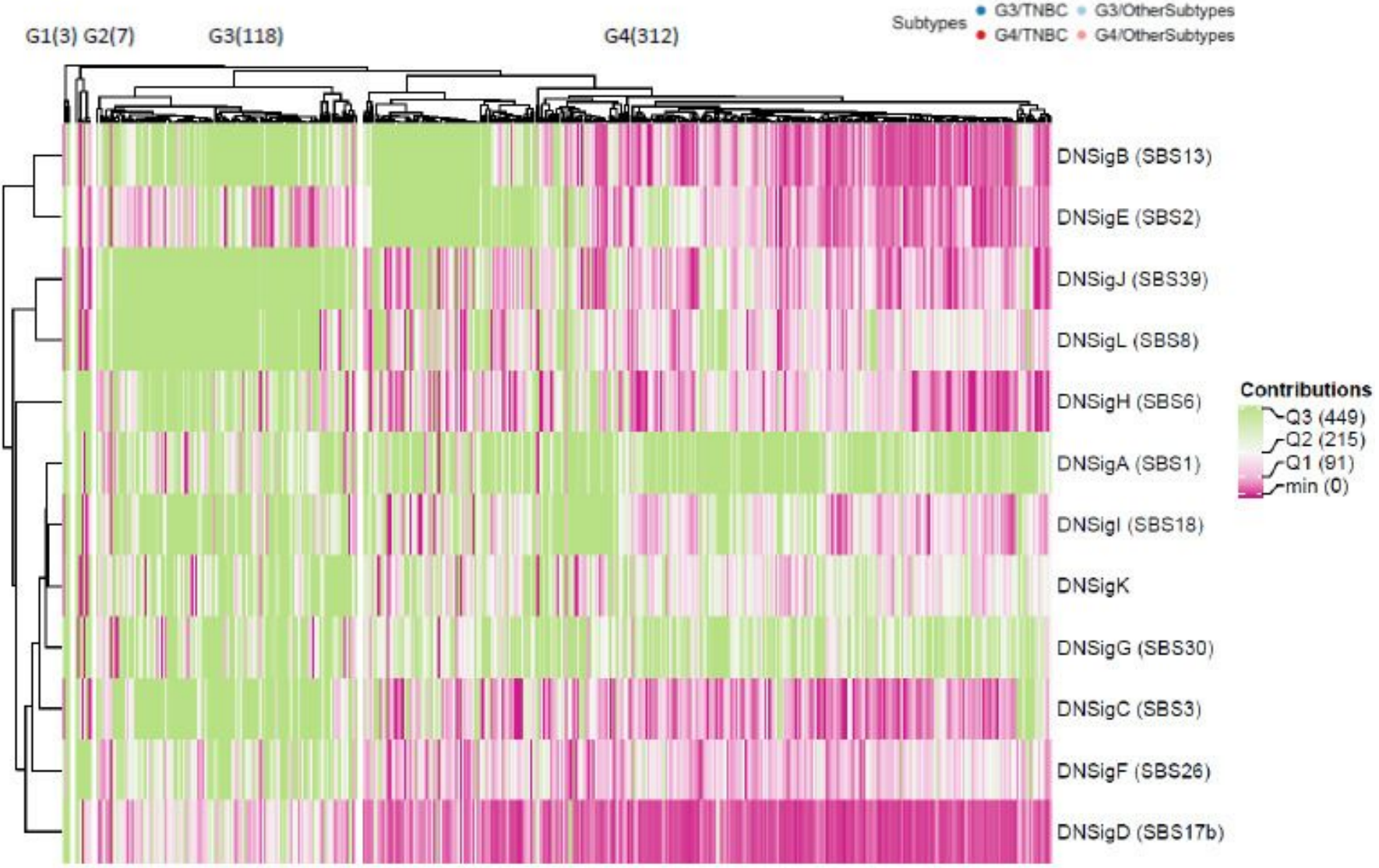

Figure 6

The results of Sanger breast cancer study by SUITOR. a) The heatmap of cosine similarity between de novo signatures detected in PCAWG breast cancer (BRCA) study and de novo signatures in Sanger BRCA study. The signatures of Sanger BRCA study are annotated by COSMIC signatures (if cosine similarities > 0.8 ) in the parentheses. For example, DNSigA (SBS1) refers to de novo signature A (DNSigA) being annotated by COSMIC signature SBS1. b) The heatmap of signature contributions estimated by SUITOR 
with hierarchical clustering in Sanger BRCA study. The number of tumors included in each signature cluster is shown in the parentheses on the top of the heatmap. Q1, Q2, Q3: the 1st, 2nd and 3rd quantiles of signature contributions. c) The t-SNE visualization of tumors in G3 and G4 signature clusters, colorcoded by signature clusters and molecular subtypes. TNBC: triple negative breast cancer.

\section{Supplementary Files}

This is a list of supplementary files associated with this preprint. Click to download.

- Supplementarynotes.pdf

- Supplementaryfigures.pdf

- Supplementarytables.xlsx 